\title{
UNA APROXIMACIÓN DE PROPUESTA DE EVALUACIÓN A ECONOMÍAS BASADAS EN EL CONOCIMIENTO PARA PAÍSES LATINOAMERICANOS
}

\author{
TOWARDS AN EVALUATION METHODOLOGY OF THE EFFORTS TO \\ CREATE KNOWLEDGE-BASED ECONOMIES IN LATIN AMERICAN \\ NATIONS
}

\author{
Jesús Porras Serrano ${ }^{(*)}$ \\ Instituto Politécnico Nacional, México (D.F), México
}

Resumen: El objetivo es presentar un método de evaluación ad hoc a la realidad latinoamericana, en su búsqueda de Economías Basadas en el Conocimiento. Se toma a México como prototipo, para que los países latinoamericanos repliquen a sus circunstancias particulares. El fin último, es que los países de la región, encuentren consensos para proponer una metodología en común, y así cotejar los resultados a nivel país y estatal de toda Latinoamérica; que sirvan para confrontar la efectividad de sus políticas públicas. Para tal propósito, se analizan metodologías de organizaciones económicas internacionales y de la Fundación mexicana Este país.

Palabras clave: Economía Basada en el Conocimiento; Índice de Economía del Conocimiento; Indicadores de Entrada y Salida.

\begin{abstract}
We attempt to present an ad hoc evaluation methodology for the Latin America case in their search to become knowledge-based economies. Mexico is taken as a case example so that other nations may adapt this methodology to their particular circumstances. The end target is to reach a regional methodological consensus in order to make on the one hand, national and local evaluations compatible and their findings easily comparable, and on the other, to measure the effectiveness of public policies. To this end the evaluation methodologies used by international agencies and the Este País foundation are analysed.
\end{abstract}

Keywords: Knowledge-Based Economy; Knowledge Economy Index; Input-Output Indicators.

(*) Doctor, Profesor Investigador de la Escuela Superior de Economía del Instituto Politécnico Nacional; Miembro del Sistema Nacional de Investigadores (Registro 54349).E-mail: <jporrass@ipn.mx>. Recibido en: 15.07.2014, aceptado en: 28.11.2014. 


\section{INTRODUCCIÓN}

El término Conocimiento puede representar diversas cosas y ser visto desde diferentes perspectivas. Hoy en día, esta categoría ha sido incluida en la economía como uno de los ejes que contribuyen en la producción de un país. El Conocimiento empletado como factor de producción, ocupa un lugar preponderante entre la sociedad, las empresas y las naciones.

La necesidad de estudiar este nuevo paradigma no se dejó esperar y desde hace más de medio siglo, diversos economistas analizaron la dinámica del Conocimiento en la creación de bienes y servicios. Se dice que, aquellos países que sustentan su economía en la producción, difusión y uso de la información y del Conocimiento, son Economías Basadas en el Conocimiento (EBC). Aunque el concepto es relativamente joven, diversas instituciones y organismos, se han dado a la tarea de desarrollar una metodología que midiera éste acervo. El Índice de Economía del Conocimiento, es el instrumento que muestra cómo las economías crean y hacen uso de este factor.

El objetivo de esta metodología, es identificar las fortalezas y debilidades del Distrito Federal y los 31 Estados que conforman la República Mexicana, con la intensión de proponer políticas públicas que orienten al país a una EBC. Es pertinente subrayar, que los resultados obtenidos, solo son comparables hacia el interior de México. Sin embargo, la idea fundamental, es que los países latinoamericanos tomen de guía esta metodología, utilicen sus variables correspondientes y pertinentes a su realidad y la apliquen a sus respectivos países. Pero lo trascendente de todo esto, es lograr una metodología que sea el producto de consensos de los países integrantes de la región, para estar en condiciones de hacer comparativas entre países, inclusive a escala estatal; observar la efectividad de sus concernientes políticas públicas y aprovechar las experiencias de su aplicación en la región.

\section{REVISIÓN DE METODOLOGÍAS YA ESTABLECIDAS}

El Conocimiento, como factor de producción, a instado a organizaciones económicas internacionales a proponer y establecer métodos de evaluación de los países en lo referente de las EBC, aquí se despliegan algunas de ellas, así como el de la Fundación mexicana Este País (2005), quién hace una propuesta de desagregación estatal.

\subsection{INSTITUTO DEL BANCO MUNDIAL}

Desde hace poco más de una década, el Instituto del Banco Mundial (2008) desarrolló una herramienta que ayuda a los países a mejorar su capacidad de utilización y acceso al Conocimiento, con el objetivo de que éstos sean más competitivos.

El instrumento del Banco Mundial mide, evalúa y compara una EBC, con el objetivo de conocer la capacidad que tienen de los países de competir en el mercado global. La Metodología de Evaluación del Conocimiento (KAM, por sus siglas en inglés), es un indicador integrado por 148 variables, distribuidas en 83 indicadores que estiman la 
generación y difusión de Conocimiento. Del KAM derivan, el Índice de Economía del Conocimiento y el Índice de Conocimiento (KEI y KI, por sus siglas en inglés); la diferencia entre ambos índices, está en que el primero, integra el desempeño general de la economía y los cuatro pilares base, mientras que el segundo integra 3 pilares (Sistemas de Innovación, Educación y Recursos Humanos y TIC), tal como se muestra en la figura 01.
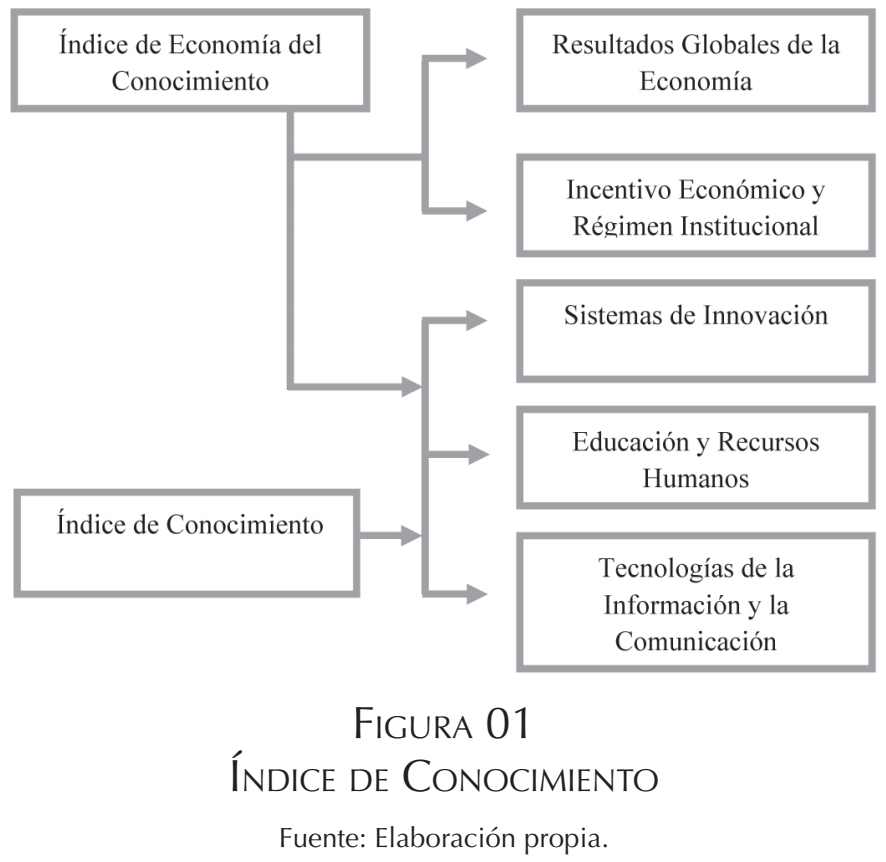

En lo Cuadro 01 (apéndices) se aprecian los 12 indicadores de los pilares y los Resultados Globales de la Economía, del KEI. El índice se construye como un promedio que adopta valores normalizados en una escala de 0 a 10, una puntuación cercana a 10 indica un desarrollo alto y una puntación más baja implica que un país se encuentra en una situación débil en comparación con otros.

\subsection{ORGANIZACIÓN PARA LA COOPERACIÓN Y EL DESARROLLO ECONÓMICO}

Así como el Banco Mundial se encargó, de construir un instrumento que midiera la cantidad de Conocimiento que genera un país, la Organización para la Cooperación y el Desarrollo Económico (OCDE), desarrolló una metodología para evaluar a una nación, en términos de una EBC.

El primer estudio de la OCDE, es la publicación The Knowledge-Based Economy en 1996, donde presentó un análisis de la dinámica de la EBC y su relación con la economía tradicional. El estudio refleja el rol que tienen el Conocimiento y la Tecnología, en el impulso de la productividad y el crecimiento económico. Las áreas clave a estimular éste crecimiento son: las inversiones en $\mathrm{I}+\mathrm{D}$, en educación y en la formación de nuevas estructuras de trabajo. 
La restructuración de los sistemas nacionales de innovación, que se componen de los flujos y las relaciones entre la industria, el gobierno y las instituciones educativas, en el desarrollo de la ciencia y la tecnología, es un determinante económico importante. El documento también toma en cuenta que, además de promover estas inversiones, se debe poner atención a la distribución de Conocimiento, en las redes formales e informales, pues son igual de importantes para el desarrollo económico.

Posteriormente, en 1999 presentó el modelo que contempla 32 indicadores distribuidos en cinco pilares, los indicadores del primer pilar se centran exclusivamente en una EBC, mientras que los restantes, comprenden las áreas denominadas: Tecnologías de Información y Comunicación; Políticas de Ciencia y Tecnología; Globalización e Impacto de la Producción (Cuadro 02 - apéndices).

Para la construcción de la metodología, la Organización se centró en un análisis profundo de las TIC, esto porque consideran, que dado el impuso y avance de estas Tecnologías, permiten el almacenamiento, el proceso y la difusión de gran cantidad de datos de forma rápida, sencilla y a bajo costo.

\subsection{FUNDACIÓN ESTE PAÍS}

La importancia que adquirió la aplicación de Conocimiento en la economía, fue notable, ahora los países centran su crecimiento, en el progreso y desarrollo de los campos que involucran a una EBC. En el país una organización no gubernamental, dedicada a la investigación y el análisis del desarrollo institucional, propuso un modelo que evalúe a México y sus entidades federativas en esta nueva economía.

La Fundación se basó en la Metodología de Evaluación del Conocimiento (MEC) del Banco Mundial (2008), adaptando los indicadores de éste último, a las variables existentes en el país; arrojando resultados para dos periodos, en el año 2005 y 2007. En lo Cuadro 03 (apéndices) se muestran los indicadores y variables de los pilares denominados, Régimen Económico e Institucional, Sistemas de Innovación, Educación y Recursos Humanos e Infraestructura de la Información, así como también, el indicador de Desempeño Económico.

La Fundación menciona, que la adaptación ilustra los espacios de crecimiento y los rezagos de las entidades federativas, para poder identificar las fortalezas y los desafíos que enfrentan para incorporarse a una EBC.

\section{PILARES DE LA METODOLOGÍA PROPUESTA}

Las metodologías anteriores son susceptibles a mejoras en dos sentidos, el primero, la desagregación de la evaluación a escala estatal; y el segundo, sustituir variables de percepción de los ciudadanos por indicadores con cierto rigor analítico.

Se procede a construir el nuevo Índice, considerando en primer término los Resultados Globales de la Economía, y posteriormente los cuatro pilares base, Incentivo Económico y Régimen Institucional, Sistemas de Innovación, Educación y Recursos Humanos, concluyendo con las Tecnologías de la Información y la Comunicación. 
En la figura 02 se puede advertir cada pilar y sus respectivas variables, la metodología propuesta integra 22 variables que se agrupan en 16 indicadores, los valores se encuentran entre 0 que es la posición más baja, y 10 el valor más alto. Se procede a abundar en cada pilar.

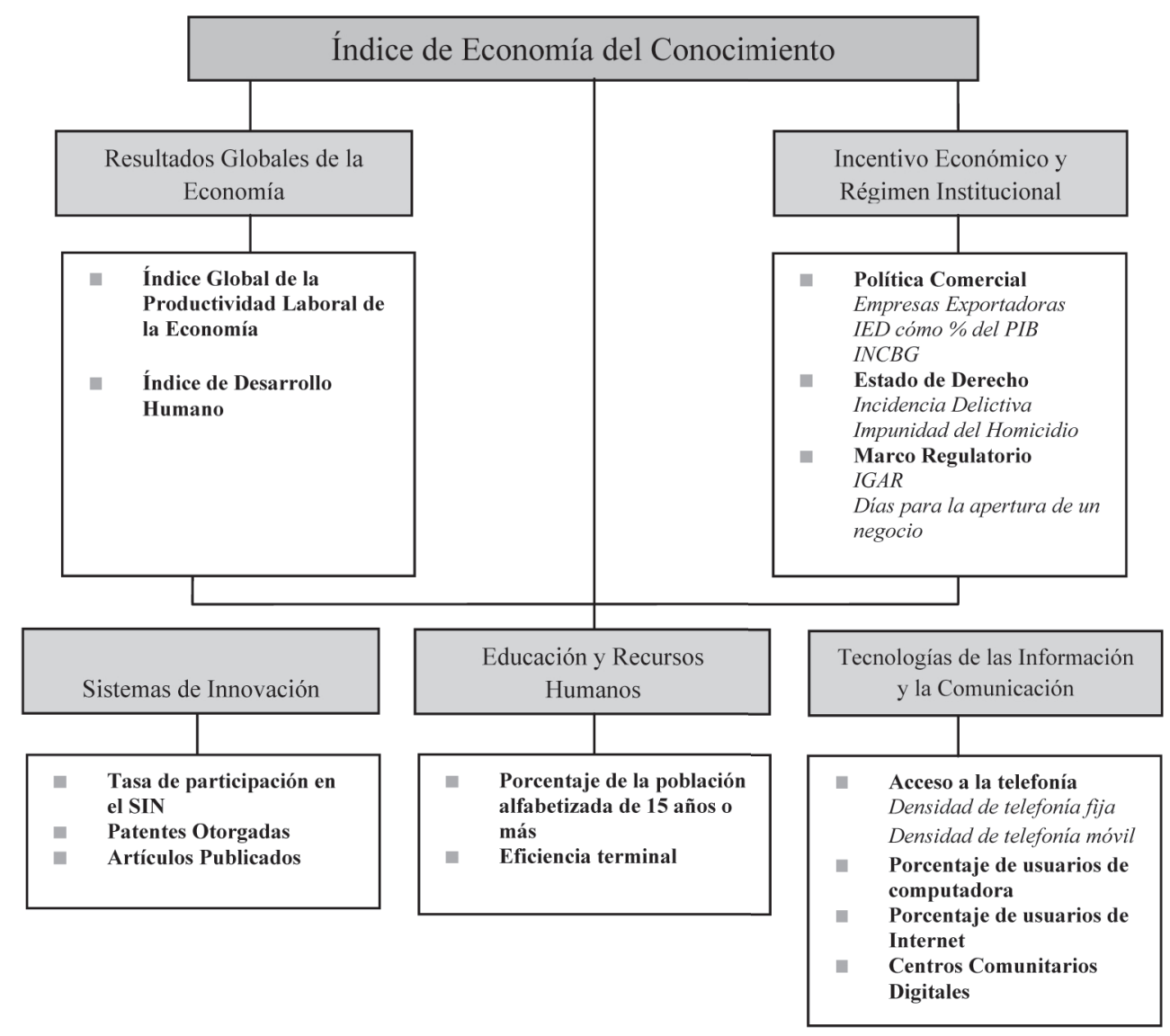

\author{
FIGURA 02 \\ Pilares de la metodoloGía Perfeccionada \\ Fuente: Elaboración propia.
}

\title{
3.1 RESUltADOS GLOBALES DE LA ECONOMÍA
}

Indicar el desempeño económico de un país es trascendental para saber la eficiencia con la que se cuenta en términos de distribución de Conocimiento, por esta razón, se han considerado dos variables para representar esta situación.

Sin lugar a duda el PIB refleja la situación económica de un país (INEGI, 2002), pero no expresa la eficiencia con la que se está llevando a cabo dicha producción; el rendimiento con el que se utilizan y combinan los factores productivos para la producción de determinada cantidad de bienes, solo se pueden determinar a través de la producti- 
vidad, en medida que se mejora el uso de los factores de producción, hay oportunidad de incrementar los ingresos de capital y así mismo, los trabajadores tendrán mejores retribuciones. Para lograr incrementar el bienestar de la población, es preciso conocer la productividad con la que viene trabajando una nación, ya que la importancia de la misma, radica en que ésta es generadora de riqueza dentro de una sociedad.

\subsection{1 ÍNDICE GLOBAL DE PRODUCTIVIDAD LABORAL DE LA ECONOMÍA}

La medición y el cálculo más aproximado que se tiene actualmente de la productividad, es el factor trabajo, estudio que realiza el Instituto Nacional de Estadística y Geografía (INEGI, 2012b), al referir el Índice Global de Productividad Laboral de la Economía (IGPLE) como el indicador más próximo para realizar dicha medición en México, basándose en la información del valor de la producción y del factor trabajo de las unidades productivas en el país.

Este índice se calcula en base a dos referentes, el número de personas ocupadas y las horas trabajadas, pero para efectos de este documento se utilizará el IGPLE con base en la población ocupada. La cuantificación del índice es un proceso que integra el Índice del PIB (IPIB) y el Índice de Población Ocupada (IPO). El IPIB se compone del volumen de PIB real trimestral y del promedio anual de PIB real del año base.

$I P I B_{n}=\frac{P I B_{n}}{P I B_{0}} * 100$

$I P I B_{n}$ Índice del PIB total a precios constantes en el trimestre $\mathrm{n}$.

$P I B_{n}$ Producto Interno Bruto total a precios constantes del año base en el trimestre $\mathrm{n}$.

$P I B_{0}$ Promedio anual de Producto Interno Bruto trimestral total en el año base.

Ahora bien, el IPO se compone de la información de la población ocupada total en un trimestre y del promedio de anual de los ocupados en el año base.

$I P O_{n}=\frac{P O_{n}}{P O_{0}} * 100$

$I P O_{n}$ Índice de Población Ocupada total en el trimestre n.

$\mathrm{PO}_{n}$ Población Ocupada total en el trimestre n.

$\mathrm{PO}_{0}$ Promedio anual de Población Ocupada total en el año base.

Con el IPIB y el IPO el IGPLEPO resulta del cociente de ambos.

$I G P L E P O_{n}=\frac{I P I B_{n}}{I P O_{n}} * 100$

$I_{\text {IFLEPO }}$ Índice Global de la Productividad Laboral de la Economía con base en la Población Ocupada en el trimestre $n$.

$I P I B_{n} \quad$ Índice del PIB real total en el trimestre $\mathrm{n}$.

$I P O_{0} \quad$ Índice de Población Ocupada total en el trimestre n. 


\subsection{2 ÍNDICE DE DESARROLLO HUMANO}

El segundo indicador que integra la evaluación de los Resultados Globales de la Economía, es el Índice de Desarrollo Humano (IDH), la importancia de éste, radica en que sintetiza tres dimensiones básicas del desarrollo humano, salud, educación y nivel de vida (PNUD, 2012). Determinado en un rango entre cero y uno, si la cifra se acerca a uno indica que un país tienen un alto desarrollo. Cabe mencionar que a partir del informe del año 2010 se hizo una restructuración de la metodología para el cálculo del IDH, dando como resultado refinar los índices de educación e ingreso; el índice de educación busca mejorar las diferencias en la adquisición y manejo de conocimiento individual y social, mientras que el índice que mide el nivel de vida se mejoró para refleja los recursos internos de un país al emplear el Ingreso Nacional Bruto (INB) per capita.

El Índice de Salud es medido por la esperanza de vida al nacer, esto es el logro relativo de un país o entidad, respecto de la norma internacional mínima de 20 años de esperanza de vida y de una máxima de 83.4 años. Para la nueva metodología éste sigue siendo el mismo.

El Índice de Educación se obtenía de las tasas de alfabetismo y de matriculación, ahora se obtiene de la media geométrica del índice de años promedio de educación en adultos de 25 años o más y de los años de escolarización que un niño en edad de ingresar a la escuela espera recibir, en México, esto se obtiene a partir de la edad de 6 años, dado que la Secretaría de Educación Pública (Ministerio de Educación, en algunos países latinoamericanos), es la edad que se considera para iniciar la educación primaria, hasta los 24 años como promedio de término de la licenciatura o su equivalente, el resultado de los cálculos determinan los años esperados de escolaridad para menores de 25 años.

$$
I E=\frac{\sqrt{I A P E \cdot I A E E-m_{i ́ n}}}{\text { máx-mín }}
$$

IE Índice de Educación

IAPE Índice de Años Promedio de Educación

IAEE Índice de Años Esperados de Escolarización

máx Máximos

mín Mínimos

En la metodología anterior se usaba el PIB per capita en poder de paridad de compra como indicador del nivel de vida entre las personas, para la nueva metodología, a partir de la reestructuración el Índice, es calculado con el INB per capita. Con los cambios realizados para el cálculo del IDH, éste requiere de los máximos y los mínimos, con los que compara el logro de un país en cada dimensión. El desempeño de los componentes se expresa en un rango entre 0 y 1 empleando la siguiente razón.

$$
\begin{aligned}
& I C=\frac{V E-V M i ́ n}{V M a ́ x-V M i ́ n} \\
& I C \text { Índice del Componente } \\
& \text { VE Valor Efectivo }
\end{aligned}
$$


VMín Valor Mínimo

VMáx Valor Máximo

Con anterioridad, para agregar las dimensiones se utilizaba un promedio, entre los tres rubros de los que se compone el IDH. Ahora se emplea una media geométrica, que se obtiene a partir de la interacción de cada índice para reducir el grado de sustitución entre las dimensiones.

$$
I D H=I S^{\frac{1}{3}} \cdot I E^{\frac{1}{3}} \cdot I I^{\frac{1}{3}}
$$

IDH Índice de Desarrollo Humano

IS Índice de Salud

IE Índice de Educación

II Índice de Ingreso

Es así que para efectos de esta investigación los Resultados Globales de la Economía en se medirá a través del IGPLE y del IDH con los cambios en la metodología que se introdujeron para el año 2010.

\subsubsection{INCENTIVO ECONÓMICO Y RÉGIMEN INSTITUCIONAL}

Está formado de tres indicadores, Política Comercial, Estado de Derecho y Marco Regulatorio. Juntos muestran el panorama de estímulos y facilidades para la prosperidad empresarial dentro de un país, ya que las instituciones y el desarrollo económico de la misma promoverán el buen manejo y uso eficiente del Conocimiento existente y del próximo a formarse.

\subsubsection{POLÍTICA COMERCIAL}

Este indicador incluye tres variables (SE, 2013) con las cuales es posible cuantificar la liberación comercial, las variables propuestas son el número de Empresas Exportadoras, la Inversión Extranjera Directa (IED) como proporción del PIB y el Índice Nacional de Corrupción y Buen Gobierno (INCBG).

- Empresas exportadoras: inicialmente la investigación proponía incluir el Índice de Comercio, puesto que éste refleja el grado o nivel de internacionalización que tiene una economía con el resto del mundo, pero al encontrarse con la limitación de la disponibilidad de datos en importaciones y exportaciones por entidad federativa para realizar la estimación, se consideraron las Empresas Exportadoras de productos y servicios como sustituto más aproximado del Índice de Comercio. Esta variable se consideró ya que muestra el nivel de la oferta exportadora mexicana ante el resto del mundo y el grado en que los empresarios buscan promover sus productos y servicios en otros países.

- Inversión de extranjera directa como proporción de PIB: el siguiente rubro del análisis de la apertura comercial son los flujos de IED, debido a que la relación que tiene el país receptor y el país inversor es altamente beneficiosa 
para ambos (INEGI, 2012c), en el primer caso, el país receptor tiene mayor conocimiento y acceso a la tecnología del país de origen, para el país inversor se traduce en una ventaja al tener acceso a otros mercados para poder de esta manera incrementar su competitividad, estos intercambios se convierten en el estímulo del comercio internacional.

- Índice nacional de corrupción y buen gobierno: la tercera y última variable que integra éste indicador pertenece al INCBG, éste da cuenta de las experiencias y las percepciones de la población acerca de la corrupción en torno a 35 servicios públicos como lo son el pago de predial, solicitud de una beca para pagar algún tipo de estudios, recibir correspondencia, conexión de línea telefónica, entre otros (TM, 2006). El índice cuantifica los niveles de corrupción cuando una persona realiza cierto trámite, se calcula por servicio o tomando en consideración todos los servicios, para efectos de este documento, de los 35 servicios se ha tomado el trámite, pasar sus cosas en alguna aduana, retén, garita o puerto fronterizo.

La escala que utiliza el índice para su medición se encuentra entre 0 y 100, donde el valor más cercano a 100 representa un elevado nivel de corrupción, se mide de la siguiente manera.

$$
I D H=I S^{\frac{1}{3}} \cdot I E^{\frac{1}{3}} \cdot I I^{\frac{1}{3}}
$$

El valor numérico que arroje la fórmula expresará entonces los casos en que se solicitó algún servicio público y para recibirlo, se pagó alguna cantidad o dicho de otra forma se pagó una dádiva, "mordida" en México.

\subsubsection{ESTADO DE DERECHO}

Este indicador se construye a partir de tres variables, mismas que muestran el panorama en terrenos de eficiencia que tiene el Estado, al garantizar el bienestar económico y social.

- Incidencia delictiva: un objetivo primordial del gobierno es garantizar seguridad a los habitantes de una nación, evaluar el desempeño y funcionamiento que las instituciones de seguridad pública proporcionan, permitirá identificar las áreas que se requieren reforzar o modificar para disminuir las tasas de delincuencia, por esta razón, la investigación propone incluir la incidencia delictiva por cada 100 mil habitantes, como una de las variables que permita saber cómo se encuentran las entidades federativas y la nación en este rubro.

- Impunidad del homicidio doloso: la segunda propuesta, es la impunidad de homicidios dolosos, este indicador se obtiene del promedio de estudiar los casos criminológicos denominados impunidad directa e impunidad rezagada. El primer tipo de impunidad registra los casos con condena en la primera instancia de un determinado periodo, dividido entre los delitos registrados durante ese mismo periodo. El segundo tipo de impunidad, se obtiene a partir de que la impunidad directa, no incorpora la duración de los procesos penales, tomando 
en cuenta este problema, la impunidad rezagada considera las condenas de un año con respecto a la incidencia registrada al año previo.

El objetivo de incluir esta variable para medir el Indicador Estado de Derecho, es el de reflejar la eficiencia del sistema de justicia penal en México y sus 32 entidades federativas, para lo cual, los datos estadísticos se tomaron del estudio Seguridad y Justicia Penal en los estados, de la organización México Evalúa (2012); encargada de estudiar, medir y dar monitoreo a las actividades gubernamentales para evaluar su calidad y eficiencia.

- Marco regulatorio: este indicador mide la eficiencia y el modo de operar de las instituciones de gobierno, al brindar accesibilidad en la reducción de obstáculos y costos que presentan los ciudadanos o empresas, cuando estos desean realizar alguna actividad productiva. Las variables propuestas son dos y se describen a continuación.

\section{4 ÍNDICE GLOBAL DE ADMINISTRACIÓN REGULATORIA}

Los datos se tomaron del estudio Retos y Perspectivas del Sistema de Gestión Regulatoria en las Entidades Federativas, elaborado por la Red Latinoamericana de Mejora Regulatoria y Competitividad en cooperación con la Comisión Federal de Mejora Regulatoria (CARBALLO, 2012). Se utilizó esta variable porque tiene las características necesarias que reflejan la realidad de política regulatoria. La construcción del índice fue bajo la guía de los indicadores de sistemas de gestión regulatoria creados por la OCDE, para replicarlos y adaptarlos en los gobiernos locales.

- Días para la apertura de un negocio: esta variable indica el plazo de resolución de tramites por parte de las autoridades para abrir cualquier negocio y la complejidad de hacerlo, la importancia de ésta radica en que, entre más largo sea el tiempo de apertura de un negocio, más serán los obstáculos burocráticos y legales que el emprendedor debe atravesar. La variable considera los procedimientos, tiempos y costos implicados en el lanzamiento de una sociedad comercial o industria de hasta 50 empleados, con un capital inicial de 10 veces el INB de la empresa en cuestión. La información se obtuvo de Doing Busines, un proyecto del Banco Mundial que proporciona una medición de las regulaciones para hacer negocios en 189 países y de sus respectivos gobiernos locales;

- Sistemas de innovación: este pilar vislumbra la intensidad de I + D en un país, conociendo la existencia de investigadores capaces para la generación y difusión de conocimiento, vinculado al número de patentes que den cuenta del grado de innovación que se tiene (CONACYT, 2011);

- $\quad$ Tasa de participación en el SIN: la tasa de participación de los estados en el Sistema Nacional de Investigadores (SNI) es un indicador que muestra la fortaleza del país en términos de eficiencia y calidad de investigación, esta variable es significativa porque los investigadores que tiene el país son sinónimo de generación y difusión de la ciencia entre empresas, instituciones educativas y gobierno; 
- Patentes otorgadas: las innovaciones que está generando una nación, denotan un impacto en la generación de crecimiento económico y pueden verse reflejadas a través de las patentes. Una patente es la concesión de derechos de una invención de algún producto o procedimiento, que en general, soluciona un problema con una técnica o manera diferente, pero, para que se considere invención, ésta debe cumplir determinados requerimientos. La institución que se encarga de evaluar y autorizar las solicitudes es el Instituto Mexicano de la Propiedad Industrial (IMPI), actor que identifica las principales características de las actividades de invención a nivel de países, industrias, sociedades y tecnologías, para estudiar los cambios de estructura y evolución producidos en la difusión, penetración y dependencia de la tecnología. Los datos se obtuvieron del Foro Consultivo de Ciencia y Tecnología, institución que muestra el número total de patentes que registró el IMPI por entidad federativa;

- Artículos publicados: el producto de la actividad de investigación científica y tecnológica se puede evaluar a través de los artículos publicados de todos los campos de la ciencia, este índice es el reflejo de la creación de nuevos conocimientos, cada artículo debe cumplir las exigentes y rigurosas especificaciones que marcan como requisitos instituciones académicas y editoriales, por estas razones se considera importante en la metodología. El Consejo Nacional de Ciencia y Tecnología (Conacyt), publica la producción de artículos según la residencia del autor en el Informe General del Estado de la Ciencia, la Tecnología y la Innovación, en esta propuesta se consultó el informe de 2011 y se tomaron los datos del periodo de años 2002 a 2011, esto debido a que el Conacyt únicamente ofrece esta información por entidad federativa;

- Educación y recursos humanos: un factor crucial dentro de una EBC es la caracterización de la población, porque será quien impulse el crecimiento de cualquier economía. Los habitantes de una nación deben de tener un cúmulo de conocimientos, destrezas, habilidades, y talentos que se vean reflejados en la reproducción de más conocimiento. El capital humano que posee una persona depende de la formación y el grado de educación que ésta haya recibido, una mejora en este ámbito dará como resultado un avance en los sistemas productivos y de innovación, que se traducirá en una economía desarrollada (INEGI, 2012);

- $\quad$ Porcentaje de la población alfabetizada de 15 años o más: un cambio cultural y social se puede llevar a cabo a través de la labor de alfabetización, la adquisición de habilidades de lectura y escritura deben formar parte del entorno de un individuo como de una región para el impulso de cualquier economía, estas son algunas de las razones por la que se consideró este indicador.

El pilar Educación y Recursos Humanos, incluye el porcentaje de la población alfabetizada de 15 años o más, los datos estadísticos se obtuvieron del Anuario de estadísticas por entidad federativa 2012 que publica el INEGI. Este indicador cobra enorme importancia, ya que es la base sobre la que se cimentará una economía para competir dentro de una EBC; 
- Eficiencia terminal: la educación y las competencias que el humano adquiere desde la niñez, son tan importantes como el seguirlas desarrollando a los largo de toda la etapa de escolarización por la que atraviese, la matrícula de cada nivel escolar es un indicativo de que la población está adquiriendo y haciendo uso de los servicios educativos que el gobierno ofrece, pero así como es tan importante el ingreso a cualquier institución educativa, es de igual o mayor relevancia que la matrícula que se registró al inicio del ciclo escolar de cualquier nivel, sea el mismo o no haya disminuido considerablemente, dicho de otra manera, los alumnos que iniciaron cualquier nivel de escolarización, sean los mismos que lograron concluir sus estudios con satisfacción, o bien, que la deserción escolar no sea elevada;

- Tecnologías de la información y la comunicación: en una EBC el papel que juegan las TIC es crucial tanto para la industria como para el desarrollo de la población. Las TIC y su infraestructura dan cuenta de cómo el conocimiento y la información se están difundiendo a través de un país, el desarrollo de las mismas acelera el crecimiento de las EBC, basándose en el supuesto de que estas sean utilizadas para el avance, la creación y difusión de más conocimiento. Este pilar se integra de la selección de cuadro indicadores, que a su vez uno de ellos se forma de la fusión de dos variables (INEGI, 2012a);

- Acceso a la telefonía: la unión de la densidad de telefonía fija y móvil, se ha incluido como indicador por el acceso inmediato a diversas fuentes de información, estas variables muestran su utilización por la población mexicana;

- Densidad de telefonía fija: la telefonía fija dentro de las TIC, sigue siendo un indicativo para las EBC, pese al incremento de la telefonía móvil, la penetración de este tipo de telefonía no deja de ocupar un lugar preponderante en las vías de comunicación. Hoy en día la densidad de telefonía fija, son el número de líneas telefónicas fijas por cada 100 habitantes, datos que se tomaron para cada entidad federativa, del Anuario estadístico 2012 que publica la Secretaria de Comunicaciones y Transportes (SCT, 2012);

- Densidad de telefonía móvil: la telefonía móvil en México se ha incrementado exorbitantemente en unos cuantos años, este medio de comunicación forma parte importante en la vida cotidiana de las personas, por su facilidad de uso y transporte en cualquier parte del mundo; dentro de las TIC se considera importante dado que el acceso a un teléfono móvil, da paso a la propagación tanto de información como de conocimiento de manera más rápida. La densidad de telefonía móvil se refiere al número de usuarios o de líneas del dispositivo, por cada 100 habitantes, estos datos se tomaron del Anuario estadístico 2012 de la SCT;

- $\quad$ porcentaje de usuarios de computadora: la proporción de usuarios de computadora es un indicador que estima que cantidad de habitantes respecto del total de la población, utilizan la computadora a partir de la edad de 6 años, y que además tienen los conocimientos y habilidades, para encender y realizar actividades escolares, de trabajo, como medio de comunicación o de entrete- 
nimiento de manera autónoma con una computadora. Los datos se obtuvieron de las Estadísticas sobre Disponibilidad y Uso de Tecnología de Información y comunicaciones en los Hogares (ENDUTIH) 2012, del INEGI (2012a);

- Porcentaje de usuarios de internet: los usuarios de Internet, es un indicador que muestra que porcentaje del total de la población, de seis años o más, de manera eventual o cotidiana, ha hecho uso de manera autónoma del internet, para realizar tareas escolares, actividades relacionadas con el trabajo, actividades de capacitación o formación a distancia mediante videoconferencias, y actividades de entretenimiento. Estos datos fueron obtenidos de la ENDUTIH;

- Centros comunitarios digitales: los espacios de acceso al público, que prestan servicios de informática e internet a los habitantes de una comunidad, son los Centros Comunitarios Digitales (CCDs), estos centros son importantes ya que su objetivos es acercar a la población a este tipo de tecnologías para que hagan uso de las mismas de manera cotidiana y tengan paso a diferentes tipos de información, esto con el propósito de que la ciudadanía se instruya y capacite. El gobierno federal impulsa un proyecto nombrado Sistema Nacional e-México, para el desarrollo y el impulso de las TIC en beneficio de la población, con la intención de impulsar un nuevo entorno social y económico.

\section{CÁlCULO DE IEC ADAPTACIÓN PARA MÉXICO}

Contar con una metodología que estimule a los países y a sus gobiernos estatales a colaborar en la elaboración de estrategias y políticas públicas. Esta nueva metodología, es la oportunidad de mejorar los resultados de todos los campos que la involucran, y así, fortalecer todos los sistemas que integra una economía.

La producción de Conocimiento en una sociedad y en las actividades económicas, no es una labor sencilla, por ese motivo se creó un modelo más apegado a la realidad latinoamericana, que evalúe al país y a sus entidades federativas. Lo Cuadro 04 (apéndices) muestra la propuesta, integrando cada una de las variables seleccionadas.

Se procede con el cálculo mediante la fórmula de normalización (por la disparidad de la información) de la metodología que proporciona el Programa de las Naciones Unidas para el Desarrollo (NAUMANN, 2005).

$$
\text { IEC }=\frac{\text { Valor real-valor mínimo }}{\text { Valor máximo-valormínimo }} \cdot 10
$$

Cabe destacar que para los indicadores donde se fusionan dos o tres variables, se realiza un cálculo ponderado, asignando un coeficiente de $\frac{1}{2} \mathrm{o} \frac{1}{3}$ según sea el caso. Lo Cuadro 05 (apéndices) muestra los resultados de la aplicación de la metodología propuesta para el Índice de Economía del Conocimiento, en este caso de México y sus entidades federativas, así como su posición, desde el que obtuvo el mejor desempeño, hasta el más deficiente.

De acuerdo con el cálculo de la metodología, los tres estados más destacados son el D.F., Nuevo León y Colima, el primero de ellos con un índice relativamente alto en 
comparación con la segunda y tercera posición. Las últimos tres lugares pertenecen a los estados de la región del sur y son Oaxaca, Chiapas y Guerrero, mientras que otros estados pertenecientes a la misma región como el caso de Quintana Roo, tiene casi el doble de desempeño. A nivel nacional el IEC fue inferior que el D.F., situación que indica que tanto el gobierno federal como los gobierno estatales, necesitan trabajar y mejorar cada área que compone la nueva metodología.

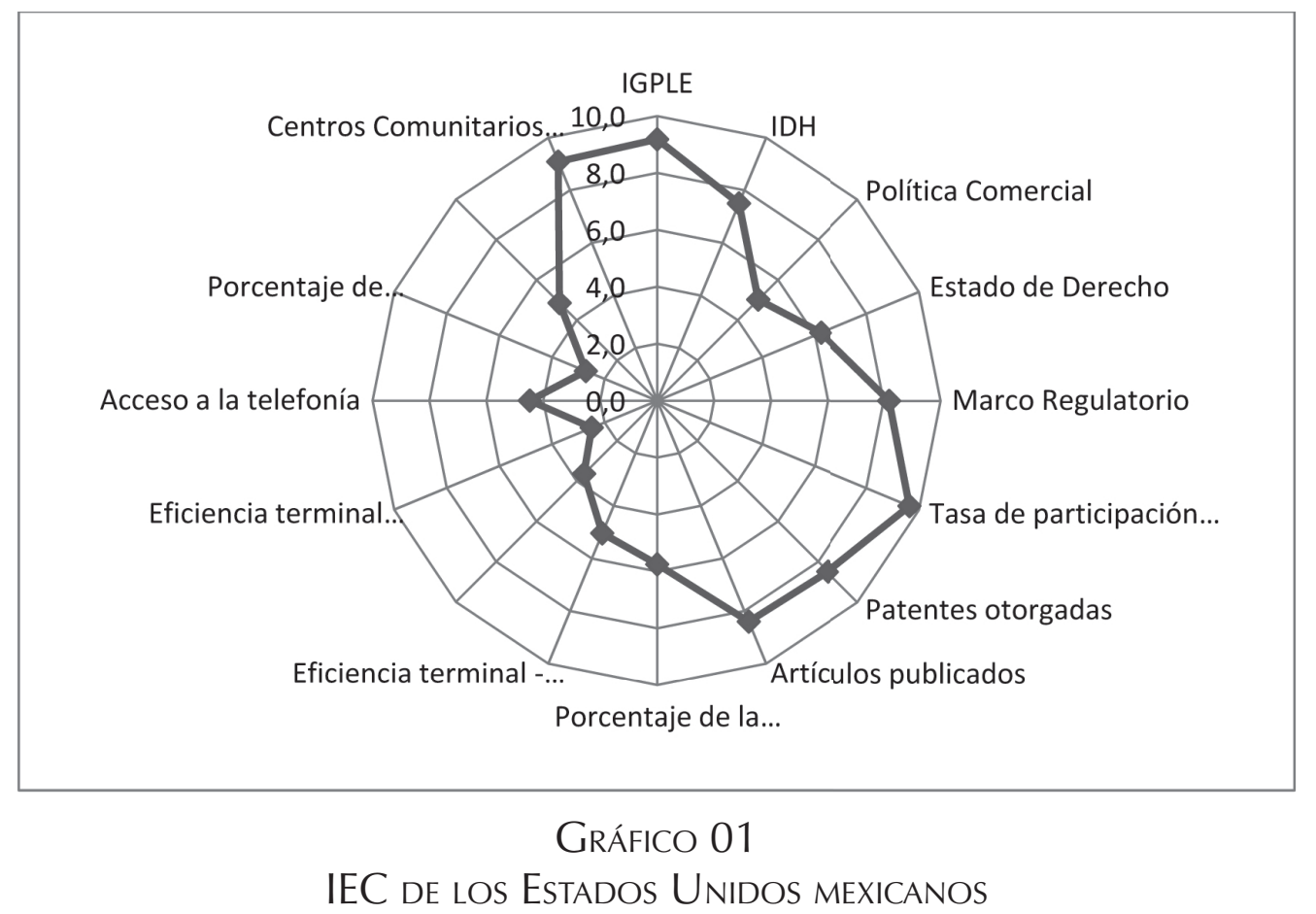

Fuente: Elaboración propia.

\section{CONCLUSIONES}

Con el método propuesto, es posible evaluar a escala estatal a diferencia de las organizaciones internacionales. Además de hacerlo con índices de comportamiento, a diferencia de la Fundación que utiliza variables de percepción.

La necesidad de crear una metodología adecuada a las condiciones de los países de la región, surge a raíz de encontrar indicadores más ad hoc a las circunstancias reales latinoamericanas que las establecidas por el Banco Mundial para evaluar una EBC. De esta forma, se pueden obtener resultados más precisos y así usar un nuevo marco metodológico en la región, que represente una oportunidad de avance para así diseñar políticas públicas que encaminen a los países latinoamericanos hacia una EBC.

La idea es incentivar a los países latinoamericanos a consensar una metodología como la presentada en el documento, con el ánimo de que a través de los resultados obtenidos, sea factible comparar entre los países, pero también, a escala estatal. El fin 
último de esta comparación, es que permita a académicos y tomadores de decisiones, observar los resultados de las políticas públicas en ambas escalas, en otras latitudes de la región y así, ver cuáles de ellas, en qué proporción y sobre todo con que adaptaciones se pueden replicar.

\section{BIBLIOGRAFÍA}

CARBALLO, Alfonso y MENDOZA, Gustavo. Retos y Perspectivas del Sistema de Gestión Regulatoria en las Entidades Federativas. México, 2012.

CONSEJO NACIONAL DE CIENCIA Y TECNOLOGÍA (CONACYT). Indicadores de Actividades Científicas y Tecnológicas. México, 2011.

FUNDACIÓN ESTE PAÍS. México ante el reto de la Economía del Conocimiento. México, 2005.

INSTITUTO NACIONAL DE ESTADÍSTICA Y GEOGRAFÍA (INEGI). El ABC de los Indicadores de la Productividad. México, 2002.

INSTITUTO NACIONAL DE ESTADÍSTICA Y GEOGRAFÍA (INEGI). Encuesta Nacional de Empleo y Ocupación 2010. México, 2011.

INSTITUTO NACIONAL DE ESTADÍSTICA Y GEOGRAFÍA (INEGI). Anuario de Estadísticas por Entidad Federativa. México, 2012.

INSTITUTO NACIONAL DE ESTADÍSTICA Y GEOGRAFÍA (INEGI). Encuesta sobre Disponibilidad y Uso de Tecnología de Información y Comunicaciones en los Hogares 2011. México, 2012, "a".

INSTITUTO NACIONAL DE ESTADÍSTICA Y GEOGRAFÍA (INEGI). Índices de Productividad Laboral y del Costo unitario de la mano de obra. México, 2012, "b".

INSTITUTO NACIONAL DE ESTADÍSTICA Y GEOGRAFÍA (INEGI). México en el Mundo 2012. México, 2012, "c".

MÉXICO EVALÚA (ME). Seguridady Justicia Penal en los Estados: 25 indicadores de nuestra debilidad institucional. México, 2012.

NAUMANN, Friedrich. México ante el reto de la Economía del Conocimiento. Resultados Nacionales y por entidad federativa. México: Fundación Este Pais/Conocimiento Util, 2005.

ORGANIZACIÓN PARA LA COOPERACIÓN Y EL DESARROLLO ECONÓMICOS (OCDE). The Knowledge-Based Economy. Francia, v. 96, n. 102, 1996.

PROGRAMA DE LAS NACIONES UNIDAS PARA EL DESARROLLO (PNUD). El Índice de Desarrollo Humano en México: cambios metodológicos e información para las entidades federativas. México. Offset Santiago. 2012.

SECRETARÍA DE COMUNICACIONES Y TRANSPORTES (SCT). Anuario estadístico 2012. México, 2012.

SECRETARIA DE ECONOMÍA (SE). Inversión Extranjera Directa en México y en el Mundo. Carpeta de Información Estadística. México, 2013.

TRANSPARENCIA MEXICANA (TM). Índice Nacional de Corrupción y Buen Gobierno. México, 2006. 


\section{APÉNDICES}

\begin{tabular}{|c|c|c|}
\hline Pilares & \multicolumn{2}{|l|}{ Indicadores } \\
\hline \multirow{2}{*}{$\begin{array}{l}\text { Resulta dos } \\
\text { Globales de } \\
\text { la Economía }\end{array}$} & \multicolumn{2}{|c|}{ Crecimiento anual del PIB. } \\
\hline & \multicolumn{2}{|c|}{ Índice de Desarrollo Humano. } \\
\hline \multirow{7}{*}{$\begin{array}{l}\text { I n c e n t i v o } \\
\text { Económico y } \\
\text { Régimen Ins- } \\
\text { titucional }\end{array}$} & \multirow{2}{*}{$\begin{array}{l}\text { Política Co- } \\
\text { mercial }\end{array}$} & Promedio de las tasas arancelarias y barreras no arancelarias. \\
\hline & & Corrupción en los servicios aduanales. \\
\hline & \multirow{3}{*}{$\begin{array}{l}\text { Estado de De- } \\
\text { recho }\end{array}$} & Percepciones de la incidencia en el crimen violento y no violento. \\
\hline & & Efectividad y predicción del Poder Judicial. \\
\hline & & Grado de cumplimiento de los contratos. \\
\hline & \multirow[t]{2}{*}{$\begin{array}{l}\text { Marco Regu- } \\
\text { latorio }\end{array}$} & $\begin{array}{l}\text { Políticas anti mercado como controles de precios o inadecuada supervisión de } \\
\text { los bancos. }\end{array}$ \\
\hline & & $\begin{array}{l}\text { Percepciones de la carga fiscal impuesta por la excesiva regulación en áreas } \\
\text { como el comercio exterior y el desarrollo del comercio. }\end{array}$ \\
\hline \multirow{3}{*}{$\begin{array}{l}\text { Sistemas de } \\
\text { Innovación }\end{array}$} & \multicolumn{2}{|c|}{ Número de investigadores en áreas de investigación y desarrollo, por millón de habitantes. } \\
\hline & \multicolumn{2}{|c|}{$\begin{array}{l}\text { Patentes otorgadas por la Oficina de Patentes y Marcas de los Estados Unidos, por millón de } \\
\text { habitantes. }\end{array}$} \\
\hline & \multicolumn{2}{|c|}{ Número de artículos científicos y técnicos publicados, por millón de habitantes. } \\
\hline \multirow{3}{*}{$\begin{array}{l}\text { Educación y } \\
\text { Recursos Hu- } \\
\text { manos }\end{array}$} & \multicolumn{2}{|c|}{ Porcentaje de la población alfabetizada, mayor de 15 años. } \\
\hline & \multicolumn{2}{|c|}{ Tasa de matriculación en educación secundaria. } \\
\hline & \multicolumn{2}{|c|}{ Tasa de matriculación en educación terciaria. } \\
\hline \multirow{3}{*}{$\begin{array}{l}\text { Tecnologías } \\
\text { de la Informa- } \\
\text { ción y la Co- } \\
\text { municación }\end{array}$} & \multicolumn{2}{|c|}{ Teléfonos por cada mil habitantes (incluye líneas telefónicas fijas y teléfonos móviles). } \\
\hline & \multicolumn{2}{|c|}{ Computadoras por cada mil habitantes. } \\
\hline & \multicolumn{2}{|c|}{ Usuarios de internet, por cada diez mil habitantes. } \\
\hline
\end{tabular}

\section{CuADro 01}

\section{INDICADORES PARA EVALUAR UNA EBC DEL BanCO MUNDIAL}

Fuente: Elaboración propia con datos del Banco Mundial.

\begin{tabular}{|c|c|}
\hline Pilares & Indicadores \\
\hline \multirow{9}{*}{$\begin{array}{l}\text { Economía Ba- } \\
\text { sada en el Co- } \\
\text { nocimiento }\end{array}$} & Inversión de Conocimiento (educación, I + D y software) como \% del PIB \\
\hline & Educación de la población adulta como \% de la población de 25-64 años \\
\hline & Gasto en I + D como \% del PIB \\
\hline & Gasto en investigación básica como \% del PIB \\
\hline & Gasto de las empresas en I + D en el Producto Interno de la Industria \\
\hline & Gasto de las empresas en I + D en la industria manufacturera \\
\hline & Participación del sector servicios en el gasto de I + D \\
\hline & Gasto en innovación como \% de las ventas totales \\
\hline & Inversión en capital de riesgo como \% del PIB \\
\hline
\end{tabular}




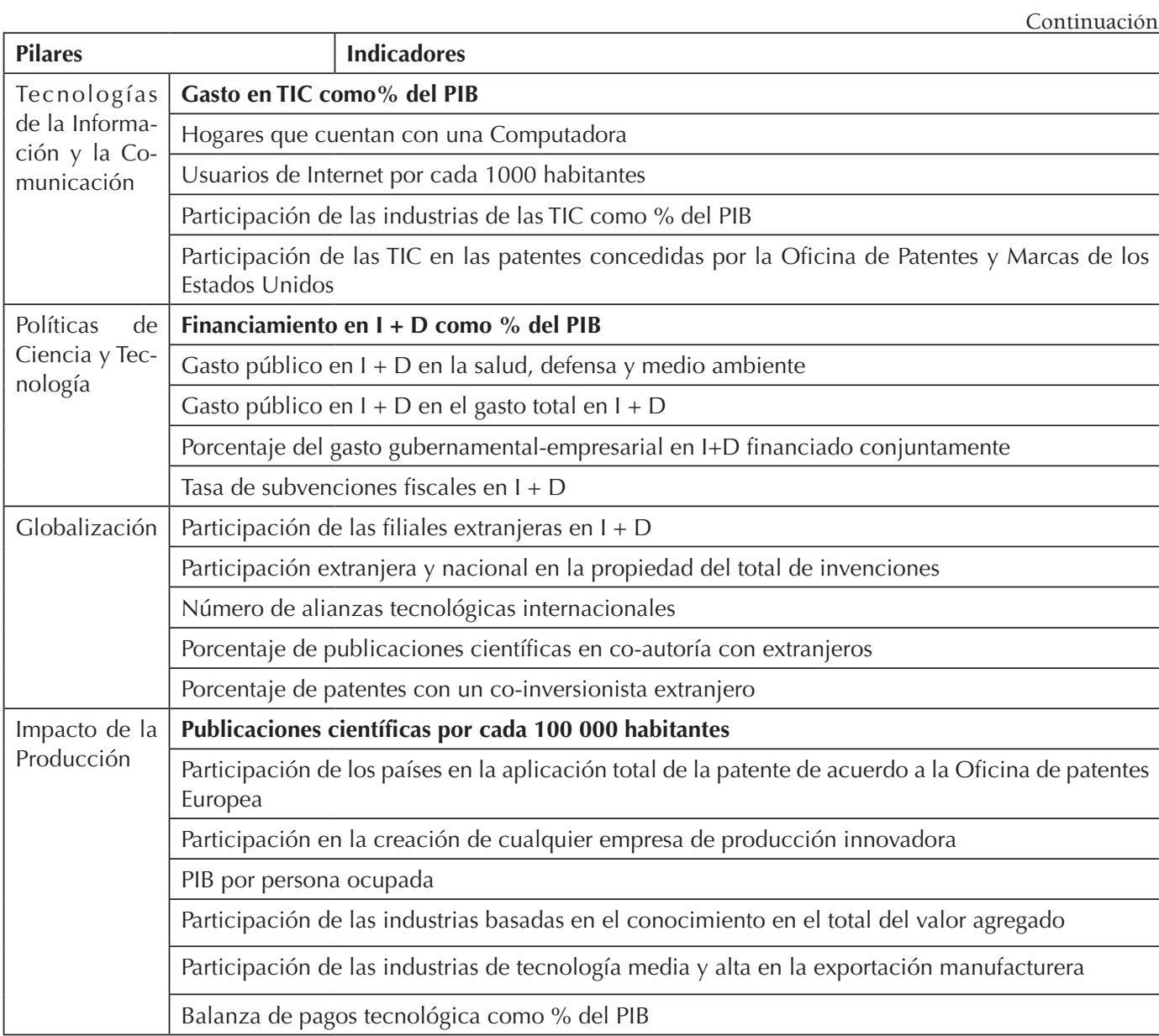

\section{Cuadro 02}

INDICADORES PARA EVALUAR UNA EBC DE LA OCDE

Fuente: Elaboración propia con datos de la OCDE.

\begin{tabular}{|c|c|c|}
\hline Pilares & \multicolumn{2}{|l|}{ Indicadores } \\
\hline \multirow{3}{*}{$\begin{array}{l}\text { Desempeño } \\
\text { Económico }\end{array}$} & \multicolumn{2}{|l|}{ Crecimiento anual del PIB } \\
\hline & \multicolumn{2}{|l|}{ Índice de Desarrollo Humano } \\
\hline & \multicolumn{2}{|l|}{ Productividad Laboral } \\
\hline \multirow[t]{3}{*}{$\begin{array}{l}\text { Régimen Ins- } \\
\text { titucional y } \\
\text { Económico }\end{array}$} & $\begin{array}{l}\text { Apertura al Exterior } \\
\text { Promedio de Inversión Extranjera Directa } \\
\text { Índice Nacional de Corrupción y Buen Gobierno }\end{array}$ & Índice de apertura \\
\hline & $\begin{array}{l}\text { Estado de Derecho } \\
\text { Proporción de delincuentes sentenciados del fuero común y del } \\
\text { fuero federal }\end{array}$ & $\begin{array}{l}\text { Porcentaje de jefes de hogar } \\
\text { que dijeron sentirse algo/muy } \\
\text { inseguros }\end{array}$ \\
\hline & $\begin{array}{l}\text { Marco regulatorio } \\
\text { Días para la apertura de un negocio }\end{array}$ & $\begin{array}{l}\text { Porcentaje de respuestas posi- } \\
\text { tivas globales por parte de los } \\
\text { empresarios }\end{array}$ \\
\hline
\end{tabular}




\begin{tabular}{|l|l|l|}
\hline \multirow{2}{*}{ Pilares } & Indicadores \\
\hline \multirow{2}{*}{$\begin{array}{c}\text { Sistemas de } \\
\text { Innovación }\end{array}$} & Número de investigadores en el Sistema Nacional de Investigadores \\
\cline { 2 - 3 } & Número de patentes solicitadas ante el Instituto Mexicano de la Propiedad Industrial \\
\cline { 2 - 3 } & Artículos científicos y técnicos publicados \\
\hline \multirow{2}{*}{$\begin{array}{l}\text { Educación yecursos Hu- } \\
\text { manos }\end{array}$} & Porcentaje de la población alfabetizada & \multicolumn{2}{|l}{} \\
\cline { 2 - 3 } & Tasa de matriculación neta en la educación secundaria & Densidad de telefonía fija \\
\cline { 2 - 3 } & Tasa de matriculación en la educación terciaria & Penetración de telefonía móvil \\
\hline \multirow{2}{*}{$\begin{array}{l}\text { Infraestructu- } \\
\text { ra de la Infor- } \\
\text { mación }\end{array}$} & Telefonía \\
\cline { 2 - 3 } & Porcentaje de viviendas particulares que disponen de computadora \\
\cline { 2 - 3 } & Centros Comunitarios Digitales \\
\hline
\end{tabular}

\section{CuAdro 03}

\section{Indicadores para eValuar una EBC de la Fundación este País}

Fuente: Elaboración propia con datos de Fundación este País (2005).

\begin{tabular}{|c|c|c|}
\hline Pilares & \multicolumn{2}{|l|}{ Metodología } \\
\hline \multirow{2}{*}{$\begin{array}{l}\text { Indicadores } \\
\text { de Resultados } \\
\text { Globales de } \\
\text { la Economía }\end{array}$} & \multicolumn{2}{|c|}{ Índice Global de Productividad laboral de la Economía } \\
\hline & \multicolumn{2}{|c|}{ Índice de Desarrollo Humano } \\
\hline \multirow{7}{*}{$\begin{array}{l}\text { Indicadores } \\
\text { de Incentivo } \\
\text { Económico } \\
\text { y Régimen } \\
\text { Institucional }\end{array}$} & \multirow{3}{*}{$\begin{array}{l}\text { Política Co- } \\
\text { mercial }\end{array}$} & Empresas Exportadoras \\
\hline & & Inversión Extranjera Directa como \% del PIB \\
\hline & & Índice Nacional de Corrupción y Buen Gobierno \\
\hline & \multirow{2}{*}{$\begin{array}{l}\text { Estado de De- } \\
\text { recho }\end{array}$} & Incidencia delictiva \\
\hline & & Impunidad del homicidio doloso \\
\hline & \multirow{2}{*}{$\begin{array}{l}\text { Marco Regu- } \\
\text { latorio }\end{array}$} & Índice Global de Administración Regulatoria \\
\hline & & Días para la apertura de un negocio \\
\hline \multirow{3}{*}{$\begin{array}{l}\text { Indicadores } \\
\text { de Sistemas } \\
\text { de Innovación }\end{array}$} & \multicolumn{2}{|c|}{ Tasa de participación en el SNI } \\
\hline & \multicolumn{2}{|c|}{ Patentes otorgadas } \\
\hline & \multicolumn{2}{|c|}{ Artículos publicados } \\
\hline \multirow{4}{*}{$\begin{array}{l}\text { Indicadores } \\
\text { de Educación } \\
\text { y Recursos } \\
\text { Humanos }\end{array}$} & \multicolumn{2}{|c|}{ Porcentaje de la población alfabetizada } \\
\hline & \multicolumn{2}{|c|}{ Eficiencia terminal - Primaria } \\
\hline & \multicolumn{2}{|c|}{ Eficiencia terminal - Secundaria } \\
\hline & \multicolumn{2}{|c|}{ Eficiencia terminal en nivel terciario } \\
\hline \multirow{5}{*}{$\begin{array}{l}\text { Indicadores } \\
\text { de Tecno- } \\
\text { logías de la } \\
\text { Información y } \\
\text { la Comunica- } \\
\text { ción }\end{array}$} & \multirow{2}{*}{$\begin{array}{l}\text { Acceso a la } \\
\text { telefonía }\end{array}$} & Densidad de telefonía fija \\
\hline & & Densidad de telefonía móvil \\
\hline & \multicolumn{2}{|c|}{ Porcentaje de usuarios de computadora } \\
\hline & \multicolumn{2}{|c|}{ Porcentaje de usuarios de internet } \\
\hline & \multicolumn{2}{|c|}{ Centros Comunitarios Digitales } \\
\hline
\end{tabular}

\section{CuAdro 04}

\section{Metodología Propuesta PARA LA EVALUACIÓN DE ECONOMÍAS BASADAS EN EL CONOCIMIENTO}

Fuente:Elaboración propia. 
Una Aproximación de Propuesta de Evaluación a Economías Basadas en el Conocimiento para Países Latinoamericanos

\begin{tabular}{|c|c|c|}
\hline \multicolumn{3}{|c|}{ Índice de Economía del Conocimiento por Entidad Federativa } \\
\hline \multicolumn{3}{|c|}{ Metodología perfeccionada 2013} \\
\hline Posición & Entidad Federativa & IEC \\
\hline 1 & Distrito Federal & 7.1659 \\
\hline 2 & Nuevo León & 5.0064 \\
\hline 3 & Colima & 4.7738 \\
\hline 4 & Sonora & 4.7207 \\
\hline 5 & Baja California Sur & 4.7008 \\
\hline 6 & Baja California & 4.4862 \\
\hline 7 & México & 4.4647 \\
\hline 8 & Jalisco & 4.3966 \\
\hline 9 & Coahuila & 4.3166 \\
\hline 10 & Morelos & 4.2338 \\
\hline 11 & Tamaulipas & 4.1577 \\
\hline 12 & Sinaloa & 4.1447 \\
\hline 13 & Quintana Roo & 4.1408 \\
\hline 14 & Querétaro & 4.1296 \\
\hline 15 & Nayarit & 4.1198 \\
\hline 16 & Aguascalientes & 4.1186 \\
\hline 17 & Hidalgo & 4.1129 \\
\hline 18 & Guanajuato & 4.1114 \\
\hline 19 & Tlaxcala & 3.9310 \\
\hline 20 & Puebla & 3.883 \\
\hline 21 & Durango & 3.807 \\
\hline 22 & Chihuahua & 3.6425 \\
\hline 23 & San Luis Potosí & 3.5957 \\
\hline 24 & Veracruz & 3.5560 \\
\hline 25 & Tabasco & 3.4487 \\
\hline 26 & Campeche & 3.4320 \\
\hline 27 & Zacatecas & 3.2960 \\
\hline 28 & Yucatán & 3.2816 \\
\hline 29 & Michoacán & 3.1130 \\
\hline 30 & Oaxaca & 2.678 \\
\hline 31 & Chiapas & 2.5839 \\
\hline \multirow[t]{2}{*}{32} & Guerrero & 2.5746 \\
\hline & Nacional & 6.2692 \\
\hline
\end{tabular}

CUADRO 05

Posición de las Entidades Federativas en el IEC

Fuente: Elaboración propia. 